\title{
Ehrenfest Theorem for the Hamilton-Jacobi Equation
}

\author{
L. KOCIS \\ Peranga Court, Unit 4, 43 Fifth Av., Sandgate QLD 4017, Australia
}

(Received July 3, 2002)

\begin{abstract}
A possible way from quantum mechanics to classical mechanics can be achieved with an exponential substitution used in the Schrödinger equation, and then considering the classical limit. This gives a picture of classical fluid and an ensemble of classical trajectories. In difference from this approach to the classical limit, while utilising the same substitution, we assume a minimum uncertainty wave packet. It is shown that this approach to the classical limit of quantum mechanics yields a single trajectory traced by the centroid of the minimum uncertainty wave packet. The momentum and the centroid of such packet satisfy the classical Hamilton-Jacobi equation.
\end{abstract}

PACS numbers: 03.65.Bz

\section{Ehrenfest theorems}

The transition from quantum mechanics to classical mechanics can be realised by means of the Eherenfest theorems

$$
\frac{\mathrm{d}}{\mathrm{d} t} \int \Psi^{*} \boldsymbol{r} \Psi \mathrm{d} V=\frac{1}{m} \int \Psi^{*}(-\mathrm{i} \hbar \nabla) \Psi \mathrm{d} V
$$

and

$$
\frac{\mathrm{d}^{2}}{\mathrm{~d} t^{2}} \int \Psi^{*} r \Psi \mathrm{d} V=\frac{1}{m} \int \Psi^{*}(-\nabla U) \Psi \mathrm{d} V
$$

For a single particle described by a wave packet, the classical limit occurs after applying two conditions [1]. These conditions are

$$
\left\langle(\Delta r)^{2}\right\rangle \rightarrow 0
$$

and

$$
\left\langle(\Delta p)^{2}\right\rangle \rightarrow 0 .
$$


For a small wave packet and for a smooth and slowly varying potential function $U$, the right side of Eq. (1.2) can be approximated

$$
-\frac{1}{m} \int \Psi^{*}[\nabla U(r)] \Psi \mathrm{d} V=-\frac{1}{m} \nabla_{\langle r\rangle} U(\langle r\rangle)
$$

Mathematical details of approximation (1.5) can be found e.g. in Refs. [1] and [2]. Utilizing (1.5) in the right side of (1.2) gives the Newton law of force for the centroid of a small wave packet

$$
\frac{\mathrm{d}^{2}}{\mathrm{~d} t^{2}}\langle r\rangle=-\frac{1}{m} \nabla_{\langle r\rangle} U(\langle r\rangle)
$$

In the classical limit the centroid of the wave packet $\langle r\rangle$ is replaced with the coordinate of the particle $\boldsymbol{r}$ and in the same way $\langle\boldsymbol{p}\rangle$ is replaced with $\boldsymbol{p}$. This leads to equations

$$
\frac{\mathrm{d} \boldsymbol{r}}{\mathrm{d} t}=\frac{1}{m} \boldsymbol{p}
$$

and

$$
\frac{\mathrm{d}^{2}}{\mathrm{~d} t^{2}} r=-\frac{1}{m} \nabla U
$$

Considering the Heisenberg uncertainty relation

$$
\left\langle(\Delta r)^{2}\right\rangle\left\langle(\Delta p)^{2}\right\rangle \geq \hbar^{2} / 4
$$

conditions (1.3) and (1.4) imply a condition

$$
\hbar \rightarrow 0 \text {, }
$$

which is generally understood as the one expressing the transition to classical mechanics.

The classical limit with conditions (1.3) and (1.4) leading to Eqs. (1.7) and (1.8) applies to the wave functions that are so-called minimum uncertainty wave packets. However, as it has been pointed out by Ballentine and collaborators, see Ref. [3], in most of the cases of the classical limit of quantum mechanics we will face problems where condition (1.10) is satisfied, but conditions (1.3) and (1.4) are not. Such circumstances signify that the classical limit of the quantum state in question will be an ensemble of classical trajectories.

\section{Hydrodynamical picture of quantum mechanics}

The classical limit of quantum mechanics with (1.10), where (1.3) and (1.4) are not applied, can be demonstrated by means of substitution

$$
\Psi=R \exp (\mathrm{i} \theta / \hbar)
$$

where $R$ and $\theta$ are real functions [4]. Substitution (2.1) applied in the time-dependent Schrödinger equation 


$$
\mathrm{i} \hbar \frac{\partial}{\partial t} \Psi=\frac{1}{2 m}(-\mathrm{i} \hbar \nabla)^{2} \Psi+U \Psi
$$

gives equations

$$
-2 m \frac{\partial R}{\partial t}=2 \nabla R \cdot \nabla \theta+R \nabla \cdot \nabla \theta
$$

and

$$
-\frac{\partial \theta}{\partial t} R=\frac{1}{2 m} R(\nabla \theta)^{2}+R U-\frac{\hbar^{2}}{2 m} \nabla \cdot \nabla R .
$$

Equation (2.4) has the form of the classical Hamilton-Jacobi equation with an additional quantum term. Equation (2.3) is equivalent to the continuity equation

$$
\frac{\partial \rho}{\partial t}+\nabla \cdot j=0
$$

where

$$
j=-\frac{\mathrm{i} \hbar}{2 m}\left(\Psi^{*} \nabla \Psi-\Psi \nabla \Psi^{*}\right)
$$

is the probability current density and the quantity

$$
\rho=\Psi^{*} \Psi
$$

is the probability density. The probability current density (2.6) and the probability density (2.7) in terms of functions $R$ and $\theta$ give

$$
j=\frac{1}{m} R^{2} \nabla \theta
$$

and

$$
\rho=R^{2}
$$

Equations (2.8) and (2.9) applied to the continuity Eq. (2.5) turn the latter into

$$
\frac{\partial\left(R^{2}\right)}{\partial t}+\nabla \cdot\left(R^{2} \frac{\nabla \theta}{m}\right)=0
$$

which is indeed the same as Eq. (2.3). The classical limit expressed with (1.10) implies that the quantum term in (2.4) is set to zero:

$$
-\frac{\partial \theta}{\partial t}=\frac{1}{2 m}(\nabla \theta)^{2}+U
$$

Realising that (2.11) has the form of the classical Hamilton-Jacobi equation and that $(2.10)$ is the continuity equation we see that $\Psi$ describes a classical fluid of non-interacting particles of mass $m$ subjected to potential $U$. The density and current density of this fluid at each point are respectively equal to the probability density and the probability current density $j$ of the quantum particle at that point. The velocity field of the classical fluid can be defined with equation

$$
v=\frac{j}{\rho}=\frac{\nabla \theta}{m} \text {. }
$$


The velocity (2.12) satisfies the law of motion of the classical fluid. To demonstrate it, $(2.12)$ is used in (2.11) to get

$$
-\frac{\partial \theta}{\partial t}=\frac{m}{2} v^{2}+U
$$

The gradient of (2.13) yields relation

$$
-m \frac{\partial v}{\partial t}=v \cdot \nabla m v+\nabla U
$$

which implies that the particles of the fluid will obey equation

$$
m \frac{\partial v}{\partial t}=-\nabla U
$$

The result (2.15) is exactly the same as (1.8), but the classical limit with the Ehrenfest theorem and the classical limit shown in this Section differ quite significantly. In Sec. 1 the Ehrenfest theorem was applied to a quantum ensemble assumed to be a minimum uncertainty wave packet. The classical limit of such quantum state is a single classical trajectory described by Eq. (1.8). The classical limit in this Section did not assume a minimum uncertainty wave packet because conditions (1.3) and (1.4) were not applied, so it yielded a velocity field and thus also an ensemble of classical trajectories commonly called "the hydrodynamical picture of quantum mechanics".

For a potential function satisfying condition $U<E$ for the whole space the hydrodynamical picture of quantum mechanics gives a spatially unrestricted velocity field, where at a given point the velocity (2.12) has exactly one value. This represents a somewhat distorted view of the reality. A realistic distribution, regardless of whether it is quantum or classical, is spatially limited and the velocity of the particle at a given point has not a single value but a distribution of different values. Studies on the time development of realistic distributions, both quantum and classical, can be found e.g. in Refs. $[3,5,6]$.

If the potential function satisfies condition $U<E$ only in a restricted area of the space, then the velocity field is also restricted into the same area. At a given point within the area $U<E$ the velocity of the particle, which is now oscillating, will have two possible values. (Particle going forth and back.) This picture contradicts the philosophy of Eq. (2.12), where a single value of the velocity is assumed. It is also at odds with any realistic case, where at a given point the velocity of the particle has to have a distribution of velocities.

\section{Hamilton-Jacobi equation for the centroid of a small wave packet}

The purpose of this Section is to show that the classical limit with substitution (2.1) does not necessarily imply the hydrodynamical picture of quantum mechanics. The approach presented here is to assume a minimum uncertainty wave packet and derive the classical Hamilton-Jacobi equation for the wave packet centroid. 
Let us compute the mean value of the $i$-th component of momentum using the formula

$$
\left\langle p_{i}\right\rangle=\int \Psi^{*}(-\mathrm{i} h) \frac{\partial \Psi}{\partial x_{i}} \mathrm{~d} V, \quad i=1,2,3 .
$$

Applying substitution (2.1) in the right side of (3.1) gives two terms

$$
\left\langle p_{i}\right\rangle=-\mathrm{i} \hbar \int R \frac{\partial R}{\partial x_{i}} \mathrm{~d} V+\int R^{2} \frac{\partial \theta}{\partial x_{i}} \mathrm{~d} V .
$$

The first integral in (3.2) is zero

$$
-\mathrm{i} \hbar \int R \frac{\partial R}{\partial x_{i}} \mathrm{~d} V=-\mathrm{i} \hbar \int \frac{\partial}{\partial x_{i}}\left(R^{2}\right) \mathrm{d} V=0
$$

because function $\Psi^{*} \Psi=R R$ is integrable. Assuming that the first derivative of $\theta$ does not change appreciably within the small wave packet, the term $\partial \theta / \partial x_{i}$ in $(3.2)$ can be replaced by $\partial \theta / \partial\left\langle x_{i}\right\rangle$. Further, the term $\partial \theta / \partial x_{i}$ in (3.2), now approximated as $\partial \theta / \partial\left\langle x_{i}\right\rangle$, can be moved in front of the integral, so the second integral in $(3.2)$ is rewritten as

$$
\int R^{2} \frac{\partial \theta}{\partial x_{i}} \mathrm{~d} V=\frac{\partial \theta\left(\left\langle x_{i}\right\rangle\right)}{\partial\left\langle x_{i}\right\rangle} \int R^{2} \mathrm{~d} V
$$

This is similar to the approximation (1.5). Equation (3.3) in (3.2) gives

$$
\left\langle p_{i}\right\rangle=\frac{\partial \theta}{\partial\left\langle x_{i}\right\rangle}
$$

The formula just obtained can be written also in the vector form

$$
\langle p\rangle=\nabla_{\langle r\rangle} \theta .
$$

The next step is to compute the dispersion of momentum. The definition of the dispersion of the $i$-th component of momentum can be written in the form

$$
\left\langle\left(\Delta p_{i}\right)^{2}\right\rangle=-\left\langle p_{i}\right\rangle^{2}+\left\langle p_{i}^{2}\right\rangle, \quad i=1,2,3 .
$$

Substitution (2.1) applied in the second term in the right side of (3.5) causes that this term expands into three integrals

$$
\begin{aligned}
& \left\langle\left(\Delta p_{i}\right)^{2}\right\rangle=-\left\langle p_{i}\right\rangle^{2}-\hbar^{2} \int R \frac{\partial^{2} R}{\partial x_{i}^{2}} \mathrm{~d} V-\mathrm{i} \hbar \int \frac{\partial}{\partial x_{i}}\left(R^{2} \frac{\partial \theta}{\partial x_{i}}\right) \mathrm{d} V \\
& +\int R^{2}\left(\frac{\partial \theta}{\partial x_{i}}\right)^{2} \mathrm{~d} V .
\end{aligned}
$$

The second integral in (3.6) is zero. The third integral in (3.6) can be approximated as $\left(\partial \theta / \partial\left\langle x_{i}\right\rangle\right)^{2}$ because of the wave packet being small, and the first term, $-\left\langle p_{i}\right\rangle^{2}$ with the help of $(3.3)$ is $-\left(\partial \theta / \partial\left\langle x_{i}\right\rangle\right)^{2}$. Thus the first term and the last term in the right side of (3.6) add up to zero. Hence for a small packet Eq. (3.6) is reduced to

$$
\left\langle\left(\Delta p_{i}\right)^{2}\right\rangle=-\hbar^{2} \int R \frac{\partial^{2} R}{\partial x_{i}^{2}} \mathrm{~d} V
$$


The vector form of $(3.7)$ is

$$
\left\langle(\Delta p)^{2}\right\rangle=-\hbar^{2} \int R \nabla \cdot \nabla R \mathrm{~d} V
$$

Now we can turn to the derivation of the classical Hamilton-Jacobi equation. The fist step in this direction was already made by applying substitution (2.1) in the Schrödinger equation (2.2) thus getting Eqs. (2.3) and (2.4). Let us multiply Eq. (2.4) by $R$ from the left and integrate over the whole space

$$
-\int \frac{\partial \theta}{\partial t} R^{2} \mathrm{~d} V=\frac{1}{2 m} \int(\nabla \theta)^{2} R^{2} \mathrm{~d} V+\int U R^{2} \mathrm{~d} V-\frac{\hbar^{2}}{2 m} \int R \nabla \cdot \nabla R \mathrm{~d} V .
$$

The result we got is exact. It was derived without neglecting anything. Note that Eq. (3.9) is like the Ehrenfest equation (1.2). Further we recall that the wave packet is small and that functions $U,(\nabla \theta)^{2}$, and $\partial \theta / \partial t$ are assumed smooth. Such functions will change only by small values within the wave packet in the same way as any reasonable and continuous function $y=f(x)$ would change within the interval $(x, x+d x)$, where $d x$ is not a differential but only a small value. At these conditions it is advantageous to assume approximations

$$
\begin{aligned}
& \int U(r) R^{2} \mathrm{~d} V=U(\langle r\rangle) \int R^{2} \mathrm{~d} V \\
& \int[\nabla \theta(\boldsymbol{r})]^{2} R^{2} \mathrm{~d} V=\left[\nabla_{\langle\boldsymbol{r}\rangle} \theta(\langle\boldsymbol{r}\rangle)\right]^{2} \int R^{2} \mathrm{~d} V
\end{aligned}
$$

and

$$
\int \frac{\partial \theta(r)}{\partial t} R^{2} \mathrm{~d} V=\frac{\partial \theta(\langle r\rangle)}{\partial t} \int R^{2} \mathrm{~d} V
$$

Approximations (3.10)-(3.12) are of the same type as approximations (3.3) and (1.5). Approximations (3.10), (3.11), and (3.12) are now utilized in (3.9) which yields equation

$$
\begin{aligned}
& \frac{-\partial \theta(\langle\boldsymbol{r}\rangle)}{\partial t} \int R^{2} \mathrm{~d} V=\frac{1}{2 m}[\nabla\langle r\rangle \theta(\langle\boldsymbol{r}\rangle)]^{2} \int R^{2} \mathrm{~d} V+U(\langle\boldsymbol{r}\rangle) \int R^{2} \mathrm{~d} V \\
& -\frac{\hbar^{2}}{2 m} \int R \nabla \cdot \nabla R \mathrm{~d} V
\end{aligned}
$$

where $\theta$ and $U$ are now functions of $\langle r\rangle$.

Function $\theta$ in Eq. (3.9) depends only on $r$ and $t$ in the same way as the wave function $\Psi$ in the Schrödinger equation (2.2) depends only on $r$ and $t$. Because of this simple fact, function $\theta$ in Eq. (3.9) does not correspond to the action function of classical mechanics. On the other hand, Eq. (3.13) has used approximations (3.10)-(3.12), where $\theta$ and $U$ became functions of $\langle r\rangle$. Looking now at (3.4) we find that for a small wave packet function $\theta$ has to be also a function of $\langle p\rangle$, and not $p$. To summarise, we state that the $\theta$ in (3.13) and (3.4) is now a function 
of $\langle p\rangle,\langle r\rangle$, and $t$, while the physical meaning of $\langle p\rangle$ is given by the Ehrenfest equation (1.1).

If we take into account that the wave function is normalised to 1 and use Eq. (3.8), Eq. (3.13) reduces to

$$
-\frac{\partial \theta(\langle\boldsymbol{r}\rangle)}{\partial t}=\frac{1}{2 m}\left[\nabla_{\langle\boldsymbol{r}\rangle} \theta(\langle\boldsymbol{r}\rangle)\right]^{2}+U(\langle\boldsymbol{r}\rangle)+\frac{1}{2 m}\left\langle(\Delta \boldsymbol{p})^{2}\right\rangle .
$$

Condition (1.4) suggests that the last term in Eq. (3.14) can be dropped. Equation (3.14) then becomes the Hamilton-Jacobi equation written for the centroid of a small wave packet

$$
-\frac{\partial \theta(\langle\boldsymbol{r}\rangle)}{\partial t}=\frac{1}{2 m}\left[\nabla_{\langle\boldsymbol{r}\rangle} \theta(\langle\boldsymbol{r}\rangle)\right]^{2}+U(\langle\boldsymbol{r}\rangle)
$$

In Eq. (3.15) function $\theta$ is now the action function of classical mechanics for $\langle p\rangle$ and $\langle r\rangle$. Because of the extremely small uncertainties of $p$ and $r$ for macroscopical bodies, we can rewrite, or rename $\langle\boldsymbol{p}\rangle$ as $\boldsymbol{p}$, and $\langle\boldsymbol{r}\rangle$ as $\boldsymbol{r}$. If we do so then (3.15) becomes (2.11) and (3.4) becomes

$$
p=\nabla \theta
$$

If potential $U$ does not depend on time, the total mean energy does not depend on time as well, and it can be calculated using formula

$$
\langle E\rangle=\int \Psi^{*} \hat{E} \Psi \mathrm{d} V
$$

where

$$
\hat{E}=\mathrm{i} \hbar \frac{\partial}{\partial t}
$$

Substitution (2.1) and the energy operator (3.18) are used in (3.17) to get

$$
\langle E\rangle=-\mathrm{i} \hbar \int R \frac{\partial R}{\partial t} \mathrm{~d} V-\int R^{2} \frac{\partial \theta}{\partial t} \mathrm{~d} V .
$$

The first integral in (3.19) is zero

$$
\int R \frac{\partial R}{\partial t} \mathrm{~d} V=\frac{1}{2} \frac{\partial}{\partial t} \int R^{2} \mathrm{~d} V=\frac{1}{2} \frac{\partial}{\partial t} \int \Psi^{*} \Psi \mathrm{d} V=0
$$

Applying (3.12) to the second integral on the right side of (3.19) gives

$$
\langle E\rangle=-\frac{\partial \theta(\langle r\rangle)}{\partial t}
$$

Equation (3.20) allows that the left side of (3.15) can be replaced by $\langle E\rangle$ 


$$
\langle E\rangle=\frac{1}{2 m}\left[\nabla_{\langle\boldsymbol{r}\rangle} \theta(\langle\boldsymbol{r}\rangle)\right]^{2}+U(\langle\boldsymbol{r}\rangle)
$$

Equation (3.21) is the Hamilton-Jacobi equation for the centroid of a small wave packet for a static field. Following the idea of the text after (3.15), we replace $\langle r\rangle,\langle p\rangle$, and $\langle E\rangle$ by $r, p$, and $E$, respectively to get

$$
E=-\frac{\partial \theta}{\partial t}
$$

and

$$
E=\frac{1}{2 m}[\nabla \theta]^{2}+U
$$

A generally valid equation that links the particle flow and particle density of a volume element, or, of a single particle, $j=\rho \boldsymbol{v}$ allows that for a small wave packet we can write equation

$$
\int j \mathrm{~d} V=\left(\int \rho \mathrm{d} V\right)\langle v\rangle
$$

where $\langle v\rangle$ denotes the velocity of the wave packet. If we use expression (2.8) in the left side of (3.24) and the formula for the probability density (2.9) in the right side of (3.24), the latter turns into equation

$$
\nabla_{\langle\boldsymbol{r}\rangle} \theta(\langle\boldsymbol{r}\rangle)=m\langle\boldsymbol{v}\rangle
$$

Consistency of (3.25) with both (3.4) and (1.1) is obvious.

The derivation of the Hamilton-J Jacobi equation given in this Section demonstrates that substitution (2.1) can be successfully applied for the transition to classical mechanics with conditions (1.3) and (1.4). The approach to the classical limit presented in this Section is so efficient that it is applicable also to the Klein-Gordon equation [7], where other approaches to the classical limit fail. The similarity of the classical limit given in this Section with the Ehrenfest theorem in Sec. 1 can be seen if we compare the Ehrenfest equation (1.2) with Eq. (3.9), and then Eq. (1.6) with Eq. (3.15).

\section{References}

[1] D.I. Davydov, Quantum Mechanics, Pergamon, New York 1965, p. 49.

[2] D.I. Blokhintsev, Quantum Mechanics, Reidel, Dordrecht 1964, p. 102.

[3] L.E. Ballentine, Y.M. Yang, J.P. Zibin, Phys Rev. A 50, 2854 (1994).

[4] A. Messiah, Quantum Mechanics, Vol. 1, North-Holland, Amsterdam 1961, p. 222.

[5] L.E. Ballentine, J.P. Zibin, Phys. Rev. A 54, 3813 (1996).

[6] L.E. Ballentine, S.M. McRae, Phys. Rev. A 58, 1799 (1998).

[7] L. Kocis, Acta Phys. Pol. A 101, 213 (2002). 